\title{
S1 Supporting Information: \\ A Non-Canonical Interaction between Calmodulin and Calcineurin Contributes to the Differential Regulation of Plant-Derived Calmodulins on Calcineurin
}

Bin Sun ${ }^{1}$, Xuan Fang ${ }^{1}$, Christopher Johnson ${ }^{2,3}$, Garrett Hauck ${ }^{2}$, Yongjun Kou ${ }^{2}$, Jonathan P. Davis ${ }^{2}$ and Peter M. Kekenes-Huskey*,1

${ }^{1}$ Department of Cell and Molecular Physiology, Loyola University Chicago, Maywood, IL, USA 60153

${ }^{2}$ Department of Physiology and Cell Biology, The Ohio State University, Columbus, OH, USA 43210

${ }^{3}$ Department of Chemistry, Mississippi State University Starkville MS, 39759 * Corresponding author. E-mail address: pkekeneshuskey@luc.edu (P. Kekenes-Huskey) 
Table S1: All molecular dynamics simulations performed in present study

\begin{tabular}{|c|c|c|}
\hline Systems & Starting structure & Simulation length \\
\hline sCaM-1 & Rosetta modelled structure & $3 \times 2 \mu \mathrm{s}=6 \mu \mathrm{s}$ \\
\hline sCaM-4 & Rosetta modelled structure & $3 \times 2 \mu \mathrm{s}=6 \mu \mathrm{s}$ \\
\hline $\mathrm{mCaM}$ & PDB ID 4Q5U & $3 \times 2 \mu \mathrm{s}=6 \mu \mathrm{s}$ \\
\hline Isolated Distal helix (DH) & Replica-Exchange MD from sequence & $100 \mathrm{~ns}$ \\
\hline sCaM-1 + DH & $2 \mathrm{MD}$ refined sCaM-1 structures $+\mathrm{DH}$ docked at 4 sites & $2 \times 4 \times 3 \times 1.2 \mu \mathrm{s}=28.8 \mu \mathrm{s}$ \\
\hline $\mathrm{sCaM}-4+\mathrm{DH}$ & $2 \mathrm{MD}$ refined sCaM- 4 structures $+\mathrm{DH}$ docked at 4 sites & $2 \times 4 \times 3 \times 1.2 \mu \mathrm{s}=28.8 \mu \mathrm{s}$ \\
\hline $\mathrm{mCaM}+\mathrm{DH}$ & Representative structure of MD simulations of $4 \mathrm{Q} 5 \mathrm{U}+\mathrm{DH}$ docked at 4 sites & $1 \times 4 \times 3 \times 1.2 \mu \mathrm{s}=14.4 \mu \mathrm{s}$ \\
\hline $\mathrm{sCaM}-1+\mathrm{DH}+\mathrm{AID}$ & Representative sCaM-1 + DH at each site with AID fused to DH C-termini & $8 \times 1 \mu \mathrm{s}=8 \mu \mathrm{s}$ \\
\hline $\mathrm{sCaM}-4+\mathrm{DH}+\mathrm{AID}$ & Representative sCaM-4 + DH at each site with AID fused to DH C-termini & $8 \times 1 \mu \mathrm{s}=8 \mu \mathrm{s}$ \\
\hline \multirow[t]{2}{*}{$\mathrm{mCaM}+\mathrm{DH}+\mathrm{AID}$} & Representative mCaM + DH at each site with AID fused to DH C-termini & $4 \times 1 \mu \mathrm{s}=4 \mu \mathrm{s}$ \\
\hline & & total: $\sim 110 \mu \mathrm{s}$ \\
\hline
\end{tabular}

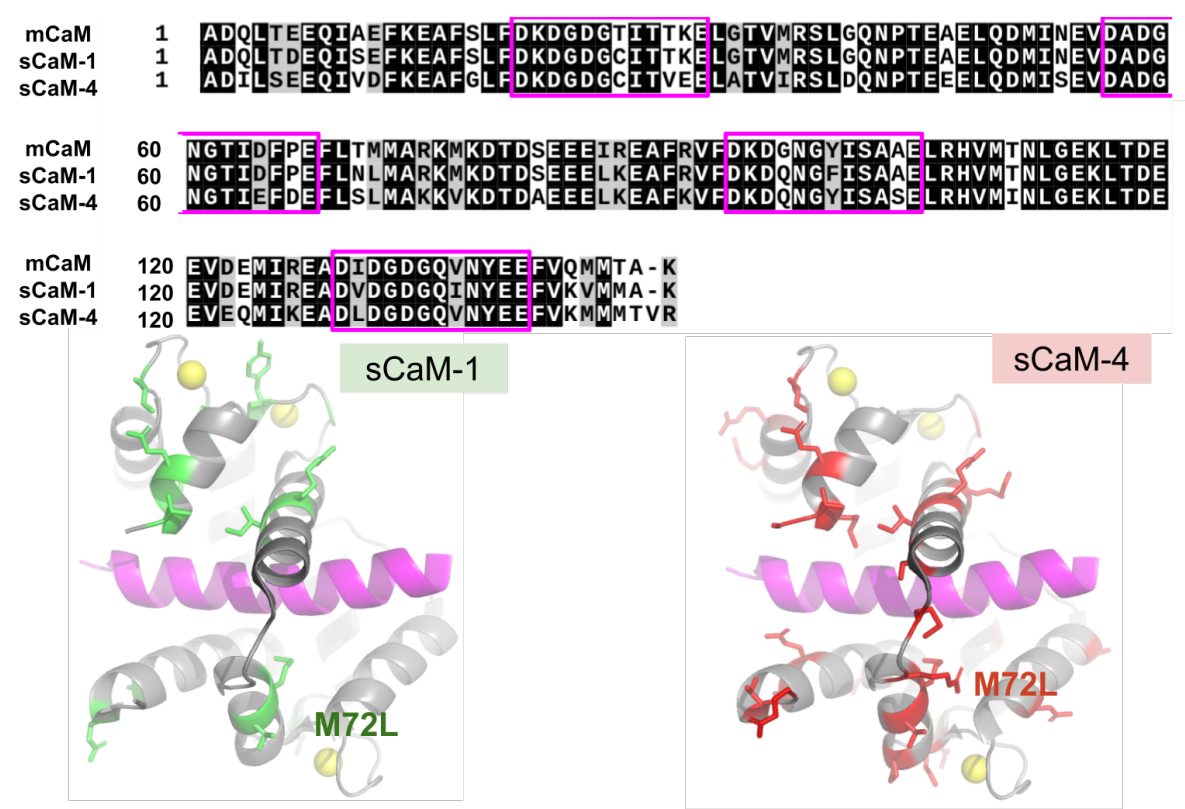

Figure S1: Sequence comparison between mammalian CaM (mCaM) and the sCaM-1/sCaM-4 soybean isoforms. The EF-hand residues are highlighted with magenta boxes. The lower panel highlights residues in soybean CaMs that are conserved in the human CaM/CaMBR complex structure (PDB 4Q5U [27]). Unique residues are shown in sticks and colored green and red for sCaM-1 and sCaM-4, respectively.

\section{S2 Supplement Method}

\section{S2.1 Protocol and flags of Rosetta Comparative Modeling}

Listing 1: HybridizeMover Protocal

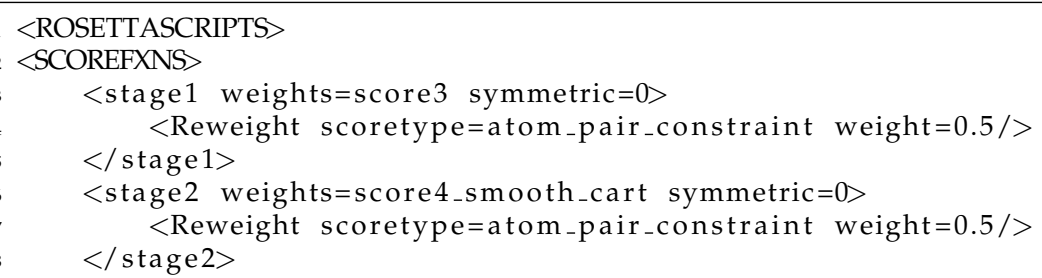




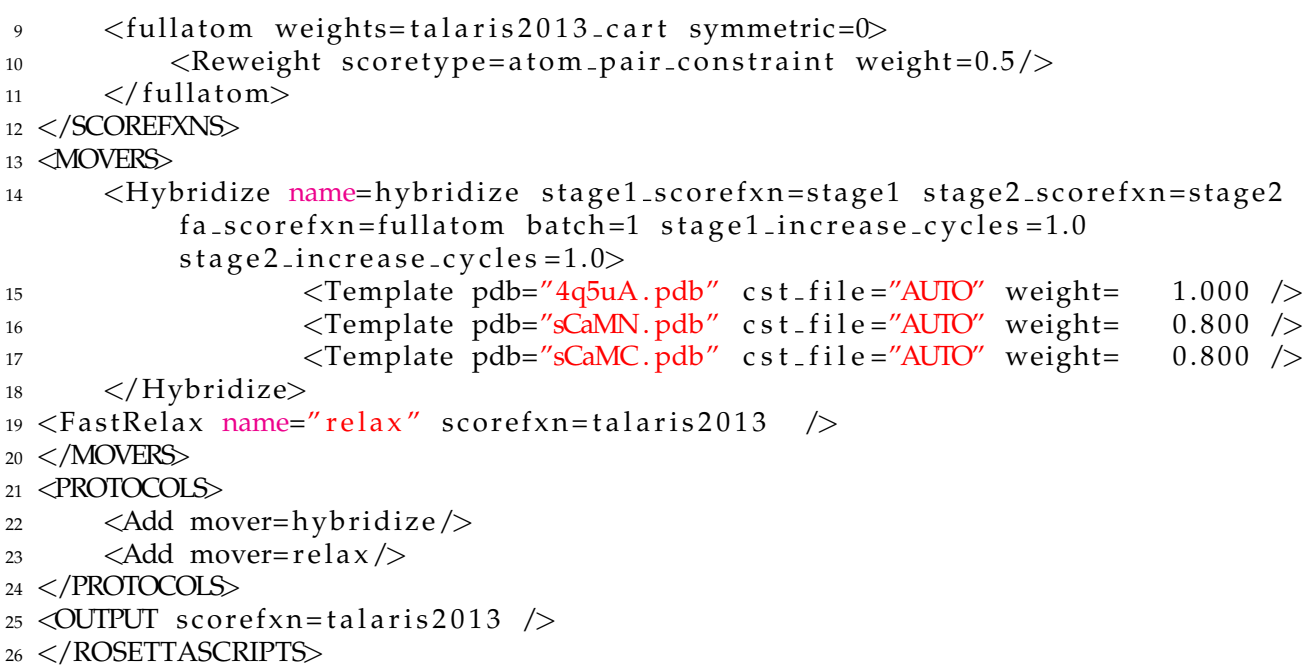

Flags:

Listing 2: Flags

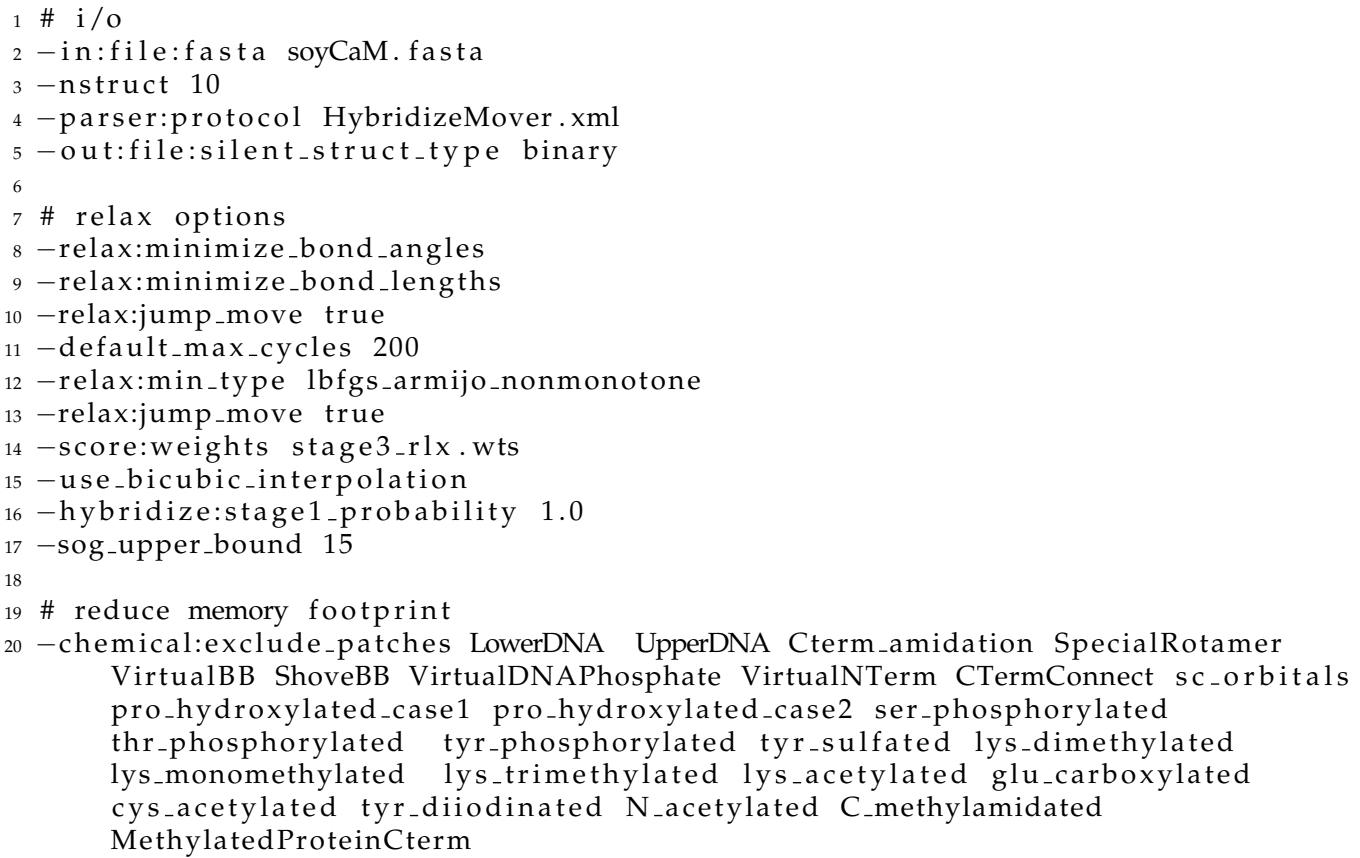




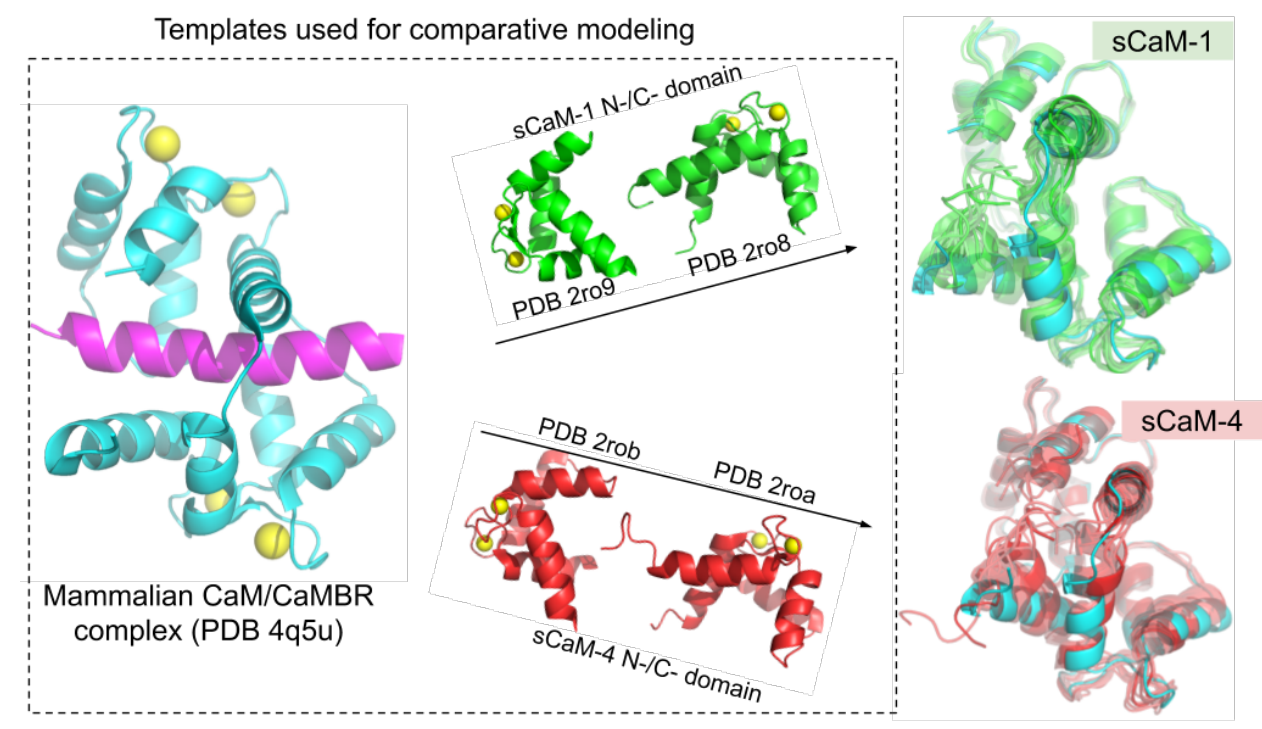

Figure S2: Rosetta comparative modeling of sCaM/CaMBR complex structures using human CaM/CaMBR crystal structure (PDB ID: 4q5u) and isolated sCaM domain structures as templates. The 10 modelled structures are superimposed on mammalian CaM template and are color green and red for sCaM-1 and sCaM-4, respectively.

\section{S2.2 Homology modeling combined with MD refinement gives reliable sCaM/- CaMBR complex structures}

Although the separate N-/C- domains structures of sCaM- 1 and sCaM-4 with $\mathrm{Ca}^{2+}$ bound in the EF-hands have been resolved [12], complete sCaM-1/4 structures complexed with CaMBR of $\mathrm{CaN}$ are not yet unavailable. We thus utilized the Rosetta comparative modeling tools [33] to build the complete structures of sCaM isofroms to provide a structural basis for exploring CaM/$\mathrm{CaN}$ interactions. Starting from the highest-scored Rosetta modeled structures, we performed 2 $\mu \mathrm{s} \mathrm{MD}$ in triplicates to refine the modeled structures as well as to investigate the structural and dynamic features of the sCaM isoforms. In addition, we also performed MD for the human $\mathrm{CaM} / \mathrm{CaMBR}$ complex starting from the crystal structure PDB 4Q5U as a comparison. 

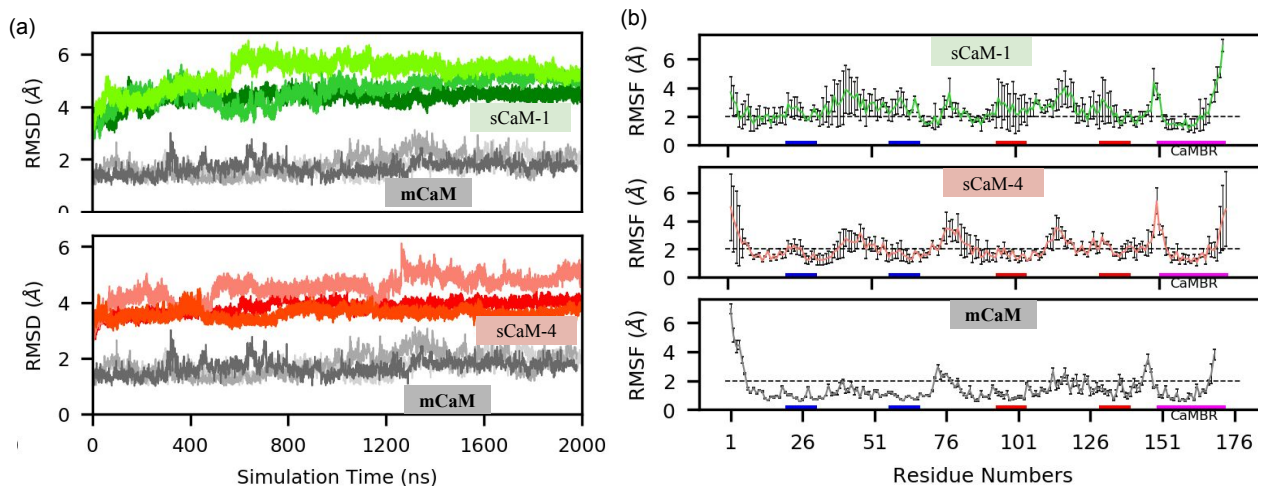

(c)

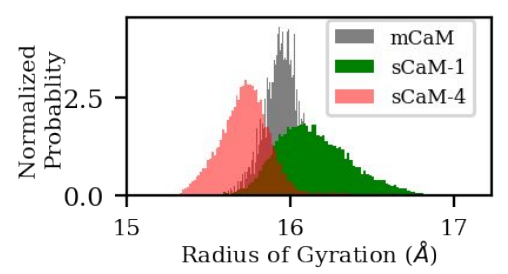

(d)
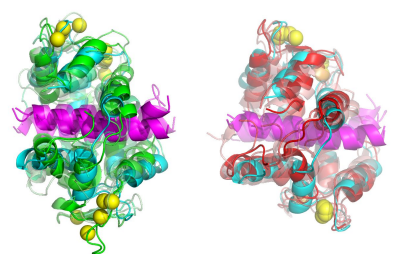

Figure S3: (a) root mean squared deviations (RMSD) of backbone atom of CaM/CaMBR. The first frame of the trajectory was used as reference of RMSD calculation. (b) root mean squared fluctuations (RMSF) of non-hydrogen atom in the triplicate MD simulations. The blue and red bars at the $\mathrm{x}$ axis represent the EF hands in the $\mathrm{N}$ and $\mathrm{C}$ domain, respectively. All frames were fitted to the first frame before RMSF calculation. The error bars are standard deviations calculated from the triplicate runs. (c) Radius of gyration distribution (d) Representative structures of sCaM1 (green) and sCaM-4 (red) from triplicate MD simulations. The human CaM/CaMBR structure (PDB 4Q5U [27]) is colored cyan with CaMBR colored magenta. $\mathrm{Ca}^{2+}$ ions are represented as yellow balls. 

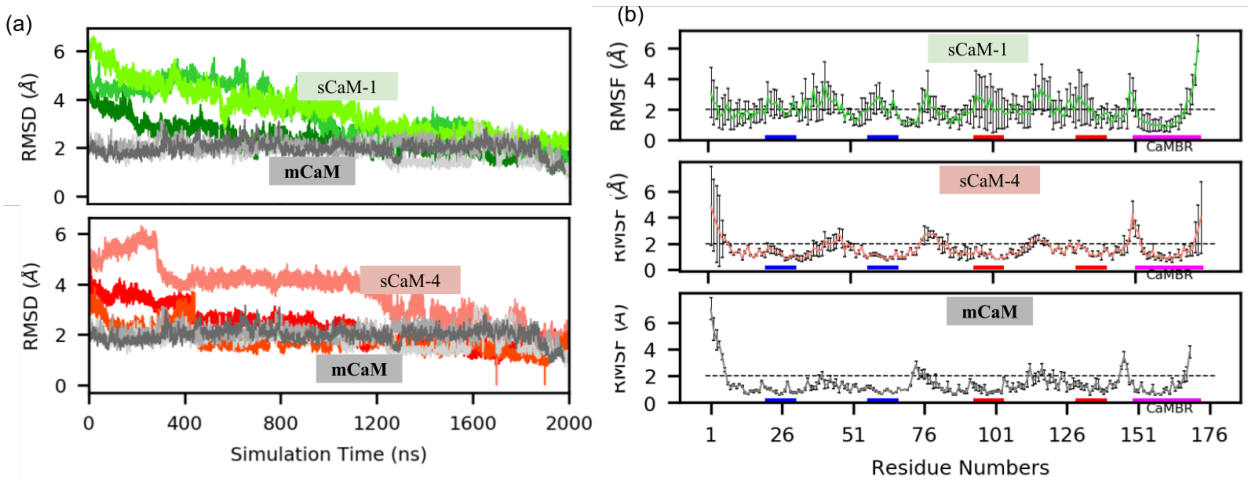

(c)

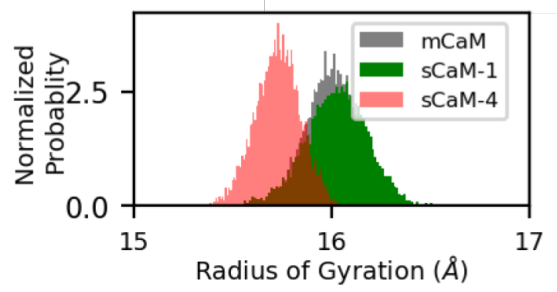

Figure S4: Same analyses as in Fig.S3 but with new data selection. The last frame of the trajectory was used as reference for RMSD calculation (a) and the last $1 \mu \mathrm{s}$ MD trajectories were used for RMSF (b) and radius of gyration (c) calculations.

As shown in Fig. S3a, the backbone atom root mean squared deviations (RMSD) values of sCaM- 1 and sCaM- 4 converges around 5 and $4 \AA$ respectively. Meanwhile, the mCaM case started from the crystal structure maintains low RMSD values around $2 \AA$. The larger RMSD values for sCaM-1 and sCaM-4 are expected and it reflects the structural relaxation of the comparative modeled structures relative to its initial structure. Despite larger RMSD values for the two sCaM isoforms, these MD refinements suggest that modeled structures are stable over the 2 $\mu$ s time-scale simulations. More importantly, electrophoresis experiments of purified sCaMs using SDS-polyacrylamide gel in the presence of $5 \mathrm{mM} \mathrm{CaCl}_{2}[6]$ has shown that sCaM- 4 has greater mobility than sCaM-1, implying that $\mathrm{Ca}^{2+}$ bound sCaM-4 is more compact. Our MD simulations support this experimental observation, as shown in Fig. S3d, sCaM-1 has significantly larger radius of gyration than sCaM-4. Experiments also show that $\mathrm{Ca}^{2+}$ binding causes more hydrophobic patch exposure in sCaM-1 than sCaM-4 as measured by 1-anilino-8-naphthalenesulfonate (ANS) fluorescence spectroscopy [12]. The less-packed hydrophobic core of sCaM-1 might be the reason why it is more extended than sCaM-4. By integration of comparative modeling with appropriately selected templates and extensive atomistic MD simulation refinement, we were able to model reliable complete sCaM structures bound with CaMBR of $\mathrm{CaN}$, as evident by 1) RMSD values that are converged over the $\mu$ s MD time-scale. Compact structure of sCaM- 4 that is consistent with experimental observations. This integrated protocol has been widely used to build protein structures that have eluded experimental structural determination [71, 72]. 

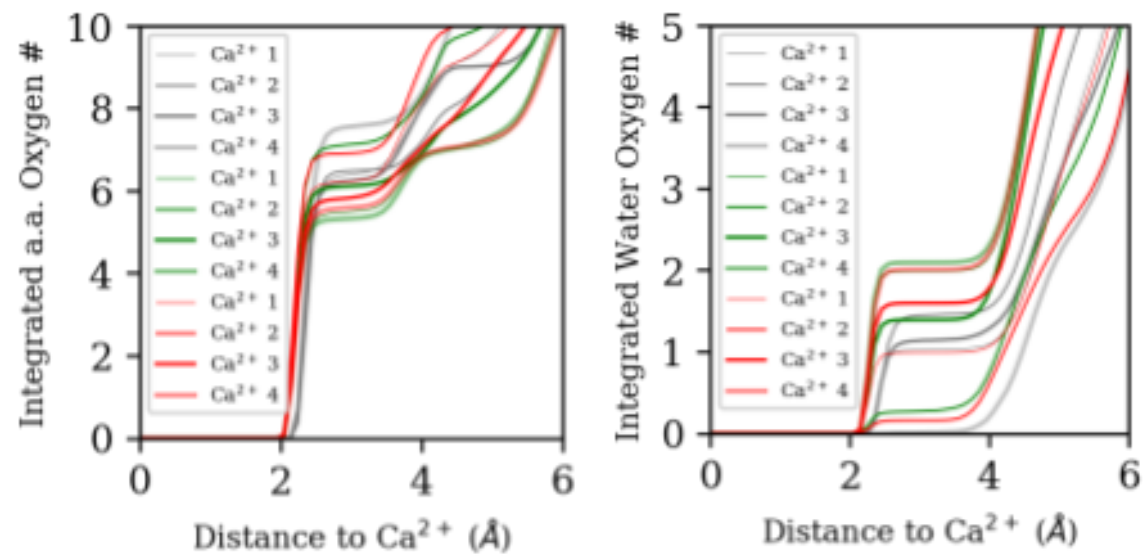

Figure S5: Integrated amino acid (a.a.,) and water oxygen numbers around $\mathrm{Ca}^{2+}$ ions.

\section{S2.3 Clustering analysis on SCaM MD trajectory to identify representative structures that will be used to dock with DH}

To perform the clustering analysis, we first divided the triplicate MD runs for each sCaM into two groups, based on the RMSD values (Fig. S6). Thus for each sCaM, we have two groups A and B. For each group, a clustering analysis were performed using the cpptraj module of Amber with the RMS of backbone atoms as distance metric. The hierarchical agglomerative (bottomup) approach was used and the MD frames were divided into ten clusters. The representative structure of the most populated cluster was picked out (the bottom panel of Fig. S6) 

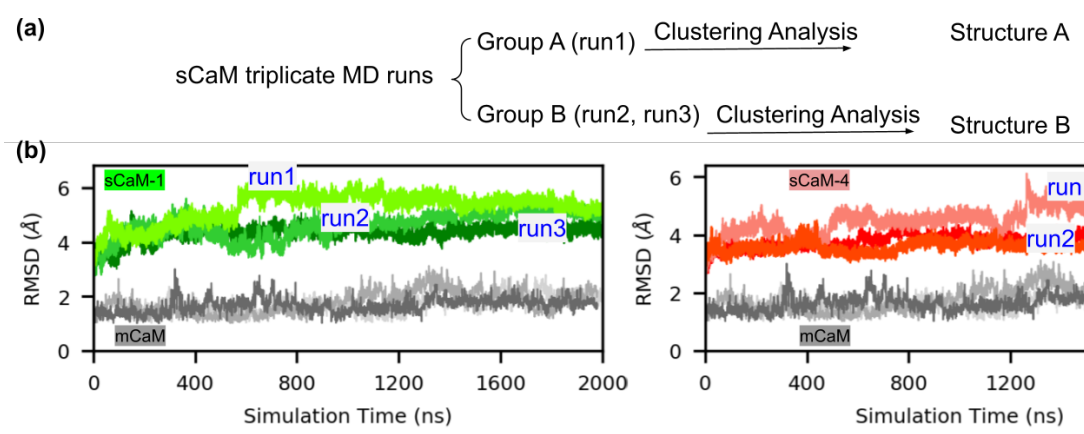

(c)
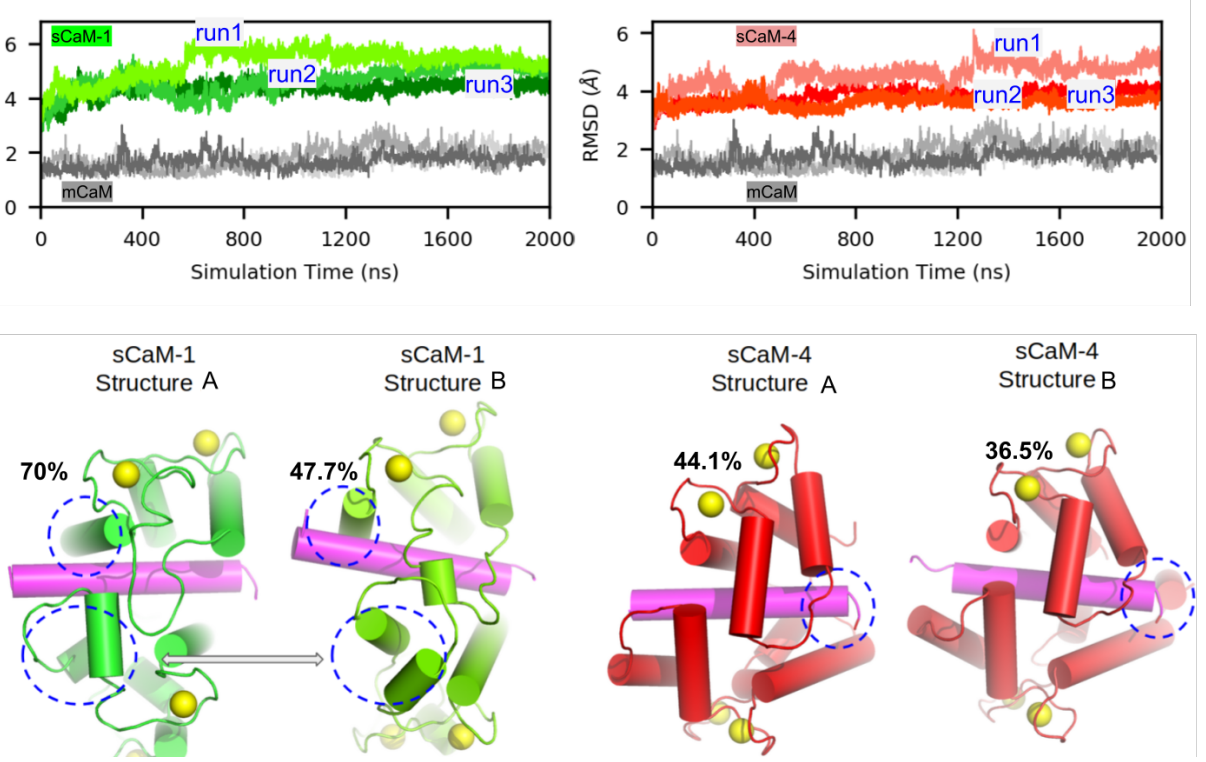

Figure S6: (a) Strategy of clustering analysis on the MD refinement trajectories. (b) Backbone atom RMSD of triplicate MD refinement simulations. The first frame was used as reference. (c) Two representative structures for each sCaM were selected to dock distal helix. The blue dashed circles highlight the structural difference of the two structures of the same sCaM.

\section{S2.4 REMD of distal helix and putative docking sites on CaM surface}




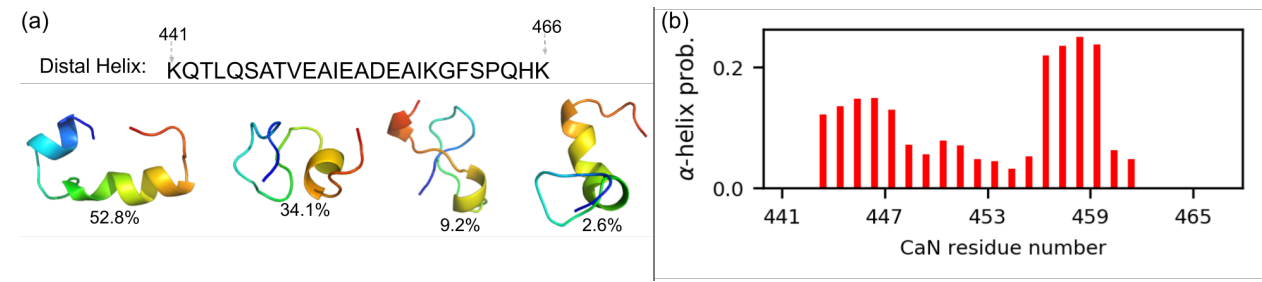

Figure S7: Structural property of distal helix sampled via $100 \mathrm{~ns}$ REMD simulation. (a) Sequence and representative structures of the first four most populated clusters. (b) $\alpha$-helix probability of each residue.

Table S2: Residues at the each tentative binding site on sCaM-1/sCaM-4 used in ZDOCK to predict distal helix interaction at each site. The slightly different residues at each site between $\mathrm{hCaM}$ and $\mathrm{sCaM}-1 / \mathrm{sCaM}-4$ was to make sure these selected residues are exposed on surface.

\begin{tabular}{cccc}
\hline Tentative Site & hCaM & sCaM-1 Residues & sCaM-4 Residues \\
\hline Site A & R86, F89, V142, Y138 & K86, R90, Y138, V142 & K86, K90, Y138, K143 \\
Site B & R106, I125, D118, D122 & R106, D118, D122, R126 & R106, D118, E122, K126 \\
Site C & I9, F12, L69, F65 & I9, K13, F65, N70 & V10, K13, F65, S70 \\
Site D & K30 ,T34, R37, Q49 & K30, V35, S38, Q49 & E30, T34, R37, Q49 \\
\hline
\end{tabular}




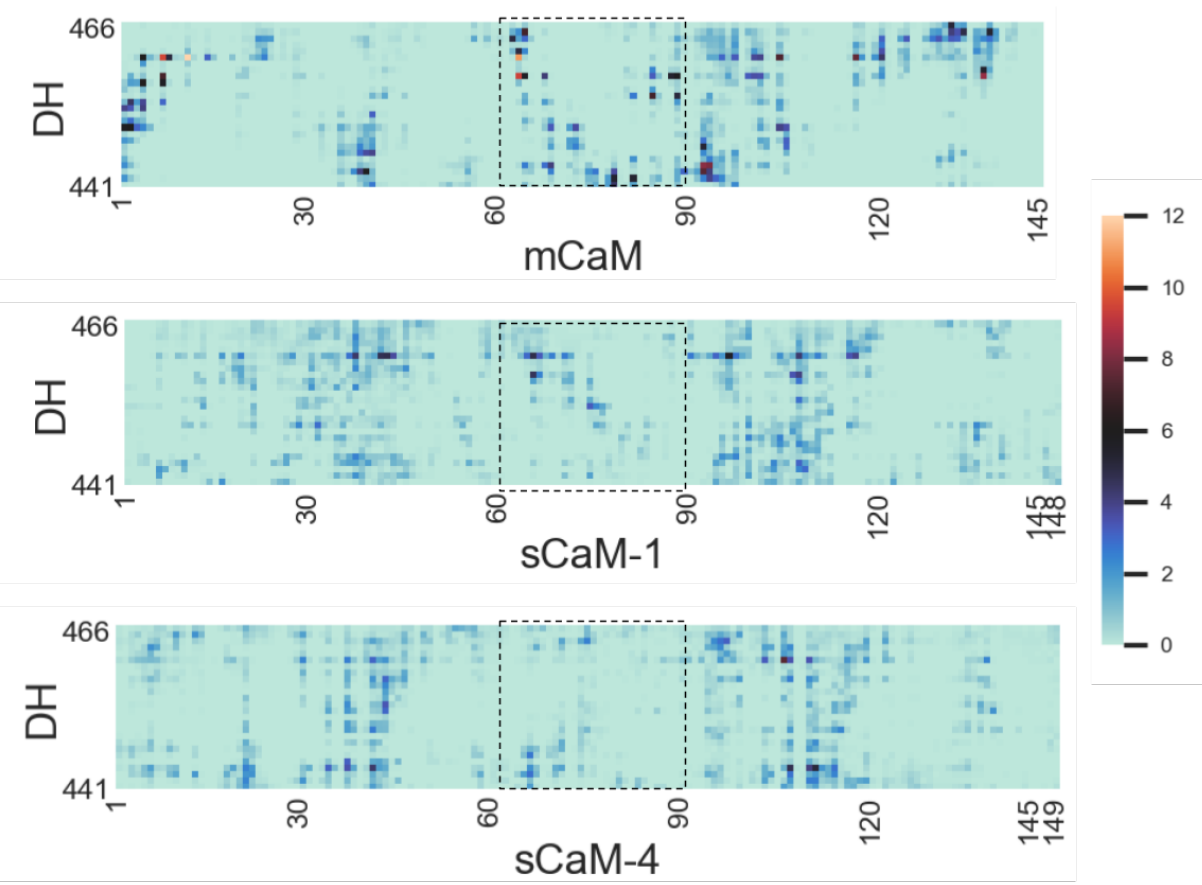

Figure S8: Contact map analysis between DH and CaM. The dashed boxes highlight the regions where sCaM- 1 and $\mathrm{mCaM}$ have similar contact that differs from sCaM-4. The contact is defined by atoms that are within $7 \AA$. All contacts from the atoms comprising the residue are summed up to serve as reside-residue contact data shown here. Thus the color bar represents the total percentages of all atoms in one residue that have contacts with other residues

\section{S2.5 Bootstrapping of the sketch-map projections to estimate the low free energy area uncertainty}

Listing 3: Pseudo code of bootstrapping

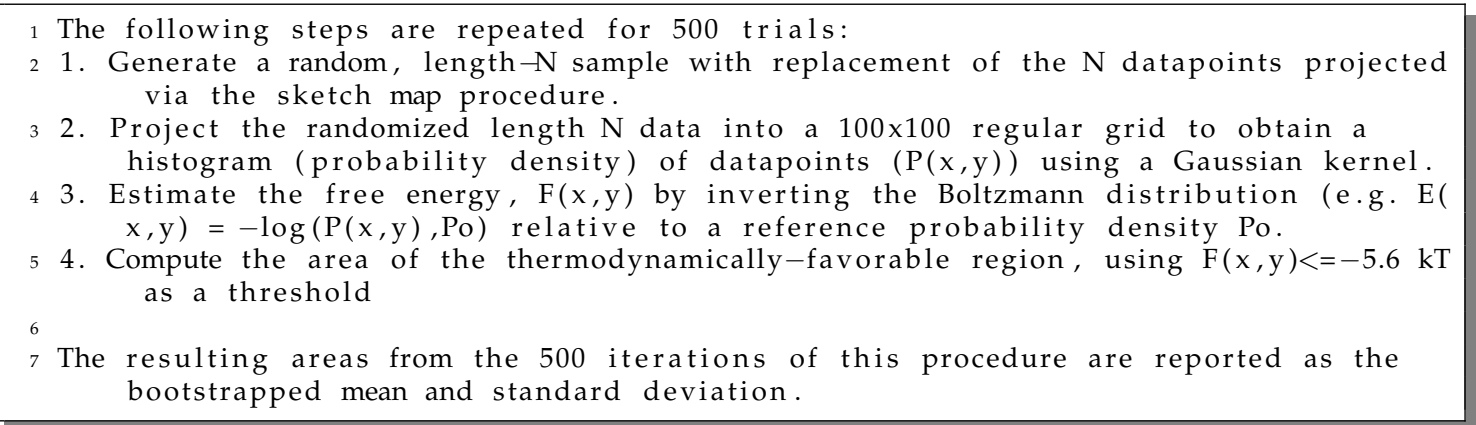

\section{S2.6 The possible consequence of the distal helix/CaM interaction can be quantitatively explained by a tether model that gives $[A I D]_{\text {eff }}$ concen- tration as the indication of $\mathrm{CaN}$ activation.}

To investigate the distal helix/CaM interaction to actual CaN activation, we utilized a tethering model our lab developed in [30] which gives the effective AID concentration ([AID] $]_{\text {eff }}$ ) near the catalytic site of CaN. Our hypothesis is that larger [AID] $]_{\text {eff }}$ leads to larger $K_{D}$ of substrate. Here we briefly summarize the key idea of the tethering model: in the absence of CaM, the regulatory domain of $\mathrm{CaN}$ is treated as a random coil and the AID is tethered by this coil (Fig. 1). 
The $[A I D]_{\text {eff }}$ around the catalytic site is determined by the length of the coil: when the length shortens, the $[\mathrm{AID}]_{\mathrm{eff}}$ is reduced near the catalytic site, which reduces competition for substrate binding. Interacting with $\mathrm{CaM}$ will shorten the length of regulatory domain as the CaMBR and distal helix regions become rigid and locked by CaM, leading to much smaller [AID] $]_{\text {eff }}$ Quantitatively, the [AID] $]_{\text {eff }}$ is given by Van Valen et al [73],

$$
[\mathrm{AID}]_{e f f}=\left(\frac{3}{4 \pi \xi L}\right)^{3 / 2} \exp \left(-\frac{3 D^{2}}{4 \xi L}\right)
$$

where $D$ depicts the distance between $\mathrm{N}$-termini of regulatory domain and catalytic site, which we assume is the distance between M387 and E481 in PDB 4OR9 [30]. $\xi$ is the persistence length determined from literature [74]. $L$ is linker length that we sampled from MD simulations. To obtain $L$ values, we started from MD refined CaM/DH complex structures and added the AID fragment to the system and performed extensive all-atom MD to sample the relative position of AID around CaM as originally described in [30]. We measured the distance between the center of mass (COM) of AID to the COM of CaM (Fig. S9a,b) and used the values as $L$ in Eq. S1. The distance distribution shows that the three CaMs have $L$ values ranging from 18 to $40 \AA$.

Putting the values in Fig. S9b as $L$ into Eq. S1, we obtained the [AID] ${ }_{\text {eff }}$ distribution for the three CaMs. The $[A I D]_{\text {eff }}$ distribution is much like the CaM-AID distance distribution. For interpretation, we assume the [AID] $]_{\text {eff }}$ value of the highest peak of $\mathrm{mCaM}$ is the critical value and the $[\mathrm{AID}]_{\mathrm{eff}} \mathrm{s}$ that are smaller and larger than this critical value will exert activating and non-activating effect on CaN, respectively (the yellow line in Fig. S9c). We calculated the areas of the activating and non-activating peaks in Fig. S9c to assess the overall effect of these CaMs on $\mathrm{CaN}$. By this measurement, we show that $\mathrm{mCaM}$ and sCaM- 1 both have $\sim 65 \%$ activating effect on CaN while sCaM-4 only has $46 \%$ (Fig. S9d). These trends suggest that sCaM-4's weaker $\mathrm{CaN}$ activating power is due to its more non-activating effect on CaN than sCaM-1 and mCaM. In other words, all CaMs are capable of activating $\mathrm{CaN}$, but sCaM-4 to a lesser extent. 